\section{Computer-Mediated Lists for Foreign Languages}

\author{
Nancy P. Shires \\ East Carolina University
}

Much is being written about the changes technology is bringing to the educational process. The whole information environment, in the present decade "is and will continue to be in a dynamic state of flux" (Kibirige, 3). This article discusses one development that modern foreign language faculty may find of use-computer-mediated discussion lists. Specifically, the article describes:

1) what lists are,

2) what their potential is, and

3) what lists are available that might be of interest to modern foreign language faculty.

\section{What are lists?}

Basically, academic lists are formed by groups of scholars who are interested in a particular subject and who communicate over computer networks. They may be referred to as "discussion lists" or "roundtable discussions" or similar terms. ${ }^{1}$ They can also be thought of as mailing lists, since messages are collected and sent out to those interested (Edmunds, 1). For example, C-18 is a list for students and scholars interested in any aspect of the eighteenth century, while LANTRA-L is a list for translators and those interested in translating. Lists areavailable through Bitnet, a telecommunications system that links academic institutions, or through a telecommunications system such as Internet, which links smaller networks together. For example, Internet can link Bitnet with other networks (Kalin and Tennant, 2). Internet is available to campuses through Bitnet and to other users through commercial services such as CompuServe. ${ }^{2}$ To receive and participate in a list, a faculty member sends an electronic message by Bitnet or Internet to the appropriate listserv address, ${ }^{3}$ many of which are described in the final section of this article. There are no membership or subscription fees, but a few lists try to limit or weed membership by requiring a special application procedure, such as submitting a resume. Most lists are edited only lightly, emphasizing the free exchange of information. A few, however, exercise heavy editing to reduce the chattiness found on popular lists. Thus, the quality of the list depends largely on the care of the participants. List memberssubmit messages (questions, ideas, opinions, facts, announcements, even first drafts of articles) to the list address. Members who call up their list mail can then see these messages and, in turn, send their own. A typical academic list might include a call for papers, a report of conference presentations, book reviews, requests for specific information, or debates about teaching methods.

Furthermore, lists are often conducted in the language of interest. For example, RUSSIAN encourages participants to correspond in Russian, and LN is primarily in French.

\section{What potential do lists have?}

Modern foreign language faculty may find lists of use in four major areas:

a) scholarly research,

b) prepublication and publication

Nancy P. Shires (M.A., Pennsylvania State University; M.L.S. Kent State University) is Assistant Professor in Academic Library Services at East Carolina University, Greenville, NC. 
c) professional networking and development, and

d) classroom activity.

\section{Scholarly Research}

Lists can function as:

- current awareness services,

- sources of highly specialized information and facts,

- places to benefit from the knowledge of experts and authorities,

- sources of information that is more recent than printed sources,

- means of clarifying knowledge,

- sources of bibliography and book reviews for professional reading or classroom use, and,

- supplements to library materials.

For example,EROFILEemphasizes book reviews of monographs in French and Italian studies and accepts backlogs of reviews from scholarly publishers. MEXICO-L concentrates on current information about Mexico which visitors might find useful.

\section{Prepublication or Publication}

Lists often furnish the opportunity to place finished book reviews and sometimes first drafts of articles. Reports of conferences written for lists can develop into publications, and certainly participation in a list can lead to requests for publication. Lists are sources of ideas for professional writing and knowledge of the appropriate markets. For example, SLART-L accepts papers from participants, so that others on the list can furnish criticism. LITERA-L aims to help its subscribers improve their writing styles.

Professional Networking and Development

Lists allow scholars to:

- find and keep in close communication with others in their area,
- arrange projects,

- make professional ties,

- test and refine ideas, and

- share ideas and concerns.

SLART-L, in its introductory message, expresses the desire to create "a community of scholars." Lists often offer the opportunity to correspond in the language being taught, thus maintaining and honing language skills.

\section{Classroom Activity}

Lists can be sources of bibliography and pedagogical ideas for the educator. For the student, they may furnish opportunities to use the target language in a real world situation or to receive current material to translate. For example, RUSSIAN aims to get students to use the Russian language.

What lists are available? ${ }^{5}$

\section{ARGENTINA}

General discussion and information about Argentina, from how to make empanadas to how to solve Argentina's problems. Includes news briefings. Mostly in Spanish.

Internet address: [ARGENTINAREQUEST@OIS.DB.TORONTO.EDU]

\section{BORIKEN}

Cultura y sociedad de Puerto Rico. Not open to automatic subscription.

Bitnet address: [LISTSERV@ENLACE or RPIRAZZ@ENLACE]

\section{C18-L}

Eighteenth century interdisciplinary discussion. "We hope to attract free-ranging discussion of topics of interest to students 
and scholars of the eighteenth century everywhere."

Bitnet address: [LISTSERV@PSUVM]

Internet address:

[LISTSERV\%PSUVM.BITNET@VM1.NODAK.EDU]

\section{CAUSERIE}

Causerie pour discuter de choses et d'autres.

Talk or chat entirely in French, for the fun of it, un Cafe Campus.

Bitnet address: [LISTSERV@UQUEBEC]

\section{CCMAN-L}

Chinese language electronic magazine.Special software needed and will be provided for a variety of terminals.

Bitnet address: [LISTSERV@UGA]

\section{CENTAM-L}

Intended as a forum for students from Central America, especially Panama and Costa Rica, and others interested for discussion of academically oriented ideas.

Bitnet address: [LISTSERV@UBVM]

Internet address:

[LISTSERV@UBVM.CC.BUFFALO.EDU]

\section{CHILE-L}

Discussion regarding Chile.

Bitnet address: [LISTSERV@USACHVM1]

Informaciones y cultura acerca de Chile.

Bitnet address: [LISTSERV@UCHCECVM]

\section{CHINA}

Discussion of any issue in Chinese studies, including but limited to anthropology, art history, economics, history, literature, linguistics, politics, religion, and sociology.

Bitnet address: [LISTSERV@PUCC]

Internet address:

[LISTSERV@PUCC.PRINCETON.EDU]

CHINA-ND

China news digest.

Bitnet address: [LISTSERV@KENTVM]

\section{CHINESE}

This list was established to promote communication among teachers, researchers, and students of Chinese.

Internet address:

[CHINESE-REQUEST@KENYON.EDU]

\section{CHINA-NN}

Daily China news network.

Bitnet address: [LISTSERV@ASUACAD]

\section{COMDEV}

Communication and international development.

Bitnet address: [COMSERVE@RPITSVM] Internet address:

[COMSERVE@VM.ITS.RPI.EDU]

\section{DANCE-L}

Global electronic forum for information exchange among all who are interested in folkdance and traditional dance. Projects include establishing an International 
Folkdance Database, a Folkdance Documentation Database, and a Multilingual Folkdance Terminology Database.

\section{Bitnet address: [LISTSERV@HEARN]}

\section{Internet address:}

[LSTSERV\%HEARNBIINET囚CUNYVMCUNYEDU]

\section{EAT-L}

Cultural food history and international recipe exchange.

Bitnet address: [LISTSERV@VTVM2]

\section{ECUADOR}

General discussion and information on Ecuador.

Internet address:

[OWNER-ECUADOR@SKAT.USC.EDU]

\section{EDUTEL}

A cross-disciplinary, cross-institutional group of scholars joined to discuss how computer-mediated communication can be used to achieve educational goals. Initial themes were how computer-mediated communication can enhance students' educational experiences within and outside the classroom; how CMC can improve the education and lifestyle of the handicapped; and how developing nations use $\mathrm{CMC}$ to foster education and research.

Bitnet address: [COMSERVE@RPITSVM] Internet address: [COMSERVE@VM.ITS.RPI.EDU]

\section{ERIC-L}

Experimental list for teaching and study of literature in education.

Bitnet address: [LISTSERV@IUBVM]
Internet address:

[LISTSERV@IUBVM.UCS.INDIANA.EDU]

\section{EROFILE}

Electronic newsletter that provides reviews of the latest books associated with French and Italian studies. Includes literary criticism, cultural studies, film studies, pedagogy, and software. Also provides a forum for comments on previously published reviews in an effort to create an on-going dialog on issues relevant to the field. Solicited and unsolicited reviews; welcomes submissions from qualified reviewers. Publishers of scholarly journals may send backlogged reviews here.

Bitnet address: [EROFILE@UCSBUXA]

Internet address:

[EROFILE@UCSBUXA.UCSB.EDU]

\section{FICINO}

Discussion of the Renaissance and Reformation.

Bitnet address: [LISTSERV@UTORONTO]

Internet address:

[LISTSERV@VM.EPAS.UTORONTO.CA]

\section{FOLKLORE}

Folklore discussion list.

Bitnet address: [LISTSERV@TAMVM1]

Internet address:

[LISTSERV@TAMVM1.TAMU.EDU]

\section{HESSE-L}

International discussion group devoted to the study of Herman Hesse and his contemporaries, including selected topics, pre-publication announcements, texts, 
interpretations, analyses, critiques, reviews, and bibliographies. In English and German.

Bitnet address: [LISTSERV@UCSBVM]

HUM3301

Modern European and Atlantic culture.

Bitnet address: [LISTSERV@UTEPA]

\section{HUMANIST}

Multidisciplinary discussion.

Bitnet address: [LISTSERV@BROWNVM]

Internet address:

[LSTSERV@BROWNVMBITNET@VMINODAKEDU]

\section{IBERIAN ISSUES}

A public bulletin board for general discussion about Latin America, Spain, and Portugal. Note,to subscribe, send email with the following message in the text field: BBOARD SUBSCRIBE IBERIAN_ISSUES <YOUR NAME $>$

Bitnet address: [NICBBS@BITNIC]

\section{I-REDES}

Spanish language networking list.

Bitnet address: [LISTSERV@UTFSM]

\section{INTERCUL}

Study of intercultural communication.

Bitnet address: [COMSERVE@RPITSVM] Internet address: [COMSERVE@VM.ITS.RPI.EDU]

\section{J-FOOD-L}

For people interested in Japanese food and culture; from Kinki University in Japan.

Bitnet address: [LISTSERV@JPNKUØ1]

Internet address: [LISTSERV\%]PNKUØ1. BITNET@CUNYVM..CUNY.EDU]

JTIT-L

Japanese teachers and instructional technology.

Bitnet address: [LISTSERV@PSUVM]

Internet address:

[LISTSERV@PSUVM.PSU.EDU]

\section{LANGUES}

Cette liste est consacrée à l'enseignement par ordinateur des secondes langues, ainsi qu'aux systèmes-experts et à l'analyse linquistique par ordinateur. Le volet INTERCULTUREL traite des rapports et des transferts culturels.

Bitnet address: [LISTSERV@UQUEBEC]

\section{LANTRA-L}

A forum for all aspects of translation and interpreting of natural languages including, but not restricted to, computer aids for translation and interpreting. For translators, educators, and others interested.

Bitnet address: [LISTSERV@SEARN]

Internet address:

[LISTSERV@SEARN.SUNET.SE]

\section{LASPAU-L}

Discussion list devoted to issues such as academic networks in Latin America and 
compilation of a directory of Latin American scholars. Also distributes information on scholarships and conferences, etc.

\section{Bitnet address: [LISTSERV@HARVARDA]}

Internet address:

[LISTSERV@HARVARDA.HARVARD.EDU]

\section{LITERA-L}

A medium for exchanging ideas about literature and related topics, such as linguistics, semantics, philology, and for sharing literary creations in order to receive constructive criticism. The list attempts some exchanging between English and Spanish literature, such as common grounds, opinions about authors, and translations.

Bitnet address: [LISTSERV@TECMTYVM]

Internet address:

[LISTSERV@TECMTYVM.MTY.ITESM.MX]

\section{LITERARY}

For those who love literature. Discussions of authors, works, styles, criticisms. For scholars and others interested.

Bitnet address: [LISTSERV@UCF1VM]

Internet address: [LISTSERV\%UCFIVM. BITNET@VM1.NODAK.EDU]

\section{LLTI}

Language learning and technology international. (See page 83 for further information.)

Bitnet address: [LISTSERV@DARTCMS1]

\section{$\mathbf{L N}$}

For computational linguists. Goal is to disseminate calls for papers, conference and seminar announcements, requests for software, corpora, and various data, project descriptions, and discussions of technical topics. Primarily in French but many items in English. Provides a forum for scholars working on French but not restricted to this field.

Bitnet address: [LISTSERV@FRMOP11]

Internet address: [LISTSERV\%FRMOP11. BITNET@VM1.NODAK.EDU]

\section{LTEST-L}

Language testing research and practice.

Bitnet address: [LISTSERV@UCLACN1]

MEXICO-D

Mexico News Distribution List

Bitnet address: [LISTSERV@TECMTYVM]

Internet address:

[LISTSERV@VMTECMEX.CEM.ITESM.MX]

\section{MEXICO-L @ TECMTYVM}

Knowing Mexico: people, places, culture. Mexico is a large and diversified county, and many of the things it has to offer are unknown to people in other parts of the world. The purpose of this list is inform people about Mexico's attractions.

Bitnet address: [LISTSERV@TECMTYVM]

Internet address:

[LISTSERV@TECMTYVM.MTY.ITESM.MX]

\section{NIHONGO}

For discussion of the Japanese language, spoken and written.

Bitnet address: [LISTSERV@MITVMA] 
Internet address: [LISTSERV@FINHUTC. BITNET@CUNYVM.CUNY.EDU]

\section{NL-KR}

Natural language and knowledge representation, including understanding, generation, recognition, parsing, semantics, pragmatics, acquisition, access, completeness.

Bitnet address: [LISTSERV@TAUNIVM] or [COMSERV@RPIECS]

Internet address:

[LISTSERV@SUNNYSIDE.COM]

\section{PERU}

General discussion, cultural exchange, news about Peru, primarily in Spanish.

Internet address:

[OWNER-PERU@CS.SFFU.EDU]

\section{RUSSIAN}

A discussion group for Russian language issues, preferably in Russian. Topics include but are not limited to Russian language, linguistics, grammar, translations, literature. This list is geared toward students, but anyone who speaks, reads, or writes Russian is encouraged to participate.

Bitnet address: [LISTSERV@ASUACAD]

Internet address:

[LISTSERV@ASUVM.INRE.ASU.EDU]

\section{SEELANGS}

Slavic and East European languages and literatures.

Bitnet address: [LISTSERV@CUNYVM]

\section{SLART-L}

A network for those involved in or interested in second or foreign language acquisition research and/or technology. Intended as a means of forming a community of scholars in SLA. Individuals may discuss research in progress, "publish" papers for feedback, solicit advice on teaching methods, etc.

Internet address:

[LISTSERV@CUNYVM.CUNY.EDU]

\section{STLHE-L}

Forum for teaching and learning in higher education. To exchange ideas, views, and experiences of importance to members of STLHE (Society for Teaching and Learning in Higher Education) and others interested. STLHE news and announcements, summaries of issues discussed in the forum.

Bitnet address: [LISTSERV@UNBVM1]

Internet address: [LISTSERV\%UNB.CA @CORNELLC.CCS.CORNELL.EDU]

\section{TEACHEFT}

Teaching effectiveness.

Bitnet address: [LISTSERV@WCU]

XCULT-L

International intercultural newsletter.

Bitnet address: [LISTSERV@PSUVM]

\section{XCULT-X}

Intercultural Communication Practicum, to foster interdisciplinary discussion of communication philosophy, theory, and practice in the global village.

Bitnet address: [LISTSERV@UMRVMB] 
Internet address:

[LISTSERV@UMRVMB.UMR.EDU]

\section{VZLA-L}

General discussion and information exchange about Venezuela, primarily in Spanish.

Bitnet address: [LISTSERV@YALEVM]

\section{Internet address:}

[LISTSERV@YALEVM.YCC.YALE.EDU]

The list above is not comprehensive and is always changing. Faculty may wish to consult their college or university libraries for further information. Forming a new list is not a difficult process. Those who do not find one that meets their needs might consider organizing a new list.

\section{NOTES}

1. They are sometimes also referred to as electronic conferences or electronic newsletters but should not be confused with interactive computer conferences or electronic newsletters or bulletin boards to which a user pays to subscribe.

2. Home computer users may be interested in reading a book such as Shapiro, Neil. The Small Computer Connection. New York: MicroText, 1983.

3. Typically the message will say SUBSCRIBE [Name of List] [Subscriber's Name], for example: SUBSCRIBE RUSSIAN Nancy Shires. Listserv is the software that processes the mail messages.

4. Pat Ensor, Bitnet communication, 26 June 1991: "Less directly, I have occasionally posted reports on Bitnet, and I always put them in a state newsletter I edit. This has led to my being offered the opportunity to do an article and a book review, since some of the people that saw these were involved with...publications."

5. Lists given here were located chiefly through the CIS List of Lists at East Carolina University and Academic Email Conferences compiled by Diane Kovacs at Kent State University. To obtain the latter, tell LISTSERV @ KENTVM to GET ACADLIST FILE1, ACADLIST FILE2, ACADLIST FILE3, ACADLIST FILE4 ACADLIST FILE5 ACADLIST FILE6.

Note: On some computers the symbol @ may be written out as the word "at."

\section{REFERENCES}

1. Edmunds, Robert A. The Prentice-Hall Encyclopedia of Information Technology. Englewood Cliffs, NJ: Prentice-Hall, 1987.

2. Kalin, Sally and Roy Tennant. "Beyond OPACS...The Wealth of Information Resources on the Internet." Database (August 1991): 28-32.

3. Kibirige, Harry M. "Information Communication Highways in the 1990s." Information Technology and Libraries 10 (1991):172.

This is an update (prepared with the assistance of Matt Carter, University of Colorado, Boulder), of an article that originally appeared in Foreign Language Annals, Volume 25, Number 6 (December 1992). Reprinted with permission of Foreign Language Annals and the American Council on the Teaching of Foreign Languages (ACTFL). 